\title{
ON A THEOREM OF OREY
}

\section{DONALD ORNSTEIN}

The starting point of this paper is the following theorem of Orey [1]: Let $T$ be a linear operator on $L_{1}$ of the integers (i.e., $T$ acts on absolutely convergent series). Assume

(a) $T$ is positive (i.e., if $f$ is a nonnegative function on the integers then so is $T f$ ),

(b) $\|T\|_{1} \leqq 1$ (i.e., $\int T f d u \leqq \int f d u$ where $u$ is the counting measure on the integers),

(c) $f \geqq 0, f \neq 0$ implies $\sum_{i=1}^{\infty} T^{i} f=\infty$ everywhere,

(d) for each pair of integers $i, j$ there is an $n_{0}(i, j)$ such that if $\delta_{i}$ is the function that is 1 on $i$ and 0 elsewhere then $T^{n} \delta_{i}(j)>0$ for all $n>n_{0}$.

Under these conditions if $f$ is a function such that $\int f d u=0$ (and $\left.\int|f| d u<\infty\right)$ then $\lim _{n \rightarrow \infty} \int\left|T^{n} f\right| d u=0$.

Sucheston began the study of operators where condition (b) is replaced by $\left(b^{\prime}\right) \sup _{n}\left\|T^{n}\right\|_{1}<\infty$. He shows, using Banach limits, that either $\lim _{n \rightarrow \infty} \int\left|T^{n} f\right| d u=0$ for all $f$ in $L_{1}$ or we can find a bounded function $e$, strictly greater than 0 , such that $T^{*} e=e .\left(T^{*}\right.$ is the adjoint of T.) (See Theorem 1, 2 in part 1 of [2]). He conjectured in a letter to the author that if $\int|f| d u<\infty$ and $\int f \cdot e d u=0$ then $\lim _{n \rightarrow \infty} \int\left|T^{n} f\right| d u=0$. The purpose of this note is to prove that conjecture.

Let $v$ be the measure induced on the integers by $e .\left(\int f d v=\int f \cdot e d u.\right)$ Then $\int f d v=\int T f d v$ and hence Orey's theorem shows that if $\int f d v=0$ then $\lim _{n \rightarrow \infty} \int\left|T^{n} f\right| d v=0$. What needs to be shown is that $\lim _{n \rightarrow \infty} \int\left|T^{n} f\right| d u=0$. (e may not be bounded away from 0 .)

LemMA. Let $u$ and $v$ be measures on the integers. Let $T$ be a linear operator satisfying (a), (c), ( $\left.\mathrm{b}^{\prime}\right)$ where $\|T\|_{1}$ is computed with respect to $u$, and (b) where $\|T\|_{1}$ is computed with respect to $v$. If $\lim _{n \rightarrow \infty} \int\left|T^{n} f\right| d v$ $=0$ then $\lim _{n \rightarrow \infty} \int\left|T^{n} f\right| d u=0$.

We will now give a proof of the above lemma. We will start with the following:

Definition. We will call a function $\tilde{g}$ a version of $g(g \geqq 0)$ if for some integer $n, \tilde{g}$ is obtained from $g$ by the following process: Let $g=g_{1}+\bar{g}_{1}$ where $g_{1} \geqq 0$ and $\bar{g}_{1} \geqq 0$ (for the rest of this definition all functions will be nonnegative). Let $T \bar{g}_{1}=g_{2}+\bar{g}_{2}$ and let $T \bar{g}_{i}=g_{i+1}+\bar{g}_{i+1}$. Let

Received by the editors September 26, 1968. 


$$
\tilde{g}=\left(\sum_{i=1}^{n} g_{i}\right)+\bar{g}_{n} .
$$

REMARKs. (A) $T^{K-1} g=\sum_{i=1}^{n} T^{K-1} g_{i}+T^{K-n} \bar{g}_{n}$ for $K>n$.

(B) If we replace any $g_{i}$ (or $\bar{g}_{n}$ ) by a version of $g_{i}$ (or $\bar{g}_{n}$ ) we still have a version of $g$.

(C) $\int g d v=\int \tilde{g} d v$.

Let $f=g-h, g \geqq 0, h \geqq 0$. It is easy to see that without loss of generality we could assume that both $g$ and $h$ are 0 for all but one integer.

(1) If $\lim _{i \rightarrow \infty}\left(\lim _{i \rightarrow \infty} \int T^{i}\left|T^{n}(g-h)\right| d u\right)=0$ then we are finished by $\left(b^{\prime}\right)$. We can therefore assume that (1) is false, i.e., there is an $\alpha>0$ such that the left side of (1) is $>\alpha$. This implies

(2) given $\epsilon$ we can find an $n$ such that $T^{n} g=r+s, r \geqq 0, s \geqq 0$, $\int r d v>(1-\epsilon) \int g d v, \lim _{i \rightarrow \infty} \int T^{i} s d u>\alpha / 2\left(r=\inf \left(T^{n} g, T^{n} h\right)\right)$. ((2) is true for $g$ or $h$ so we assume it true for $g$.)

(3) We can find a version $\tilde{r}$, of $r, \tilde{r}=r_{1}+r_{2}+\cdots+r_{m}+\bar{r}_{m}$, such that $r_{i}, i=1, \cdots, m$, is 0 or a multiple of $g$, and $\int \bar{r}_{m} d v<\epsilon \int r d v$. [(a), (b) with $u$ replaced by $v$, and (c) imply (3) by a standard argument which goes as follows: Let $j$ be the integer at which $g$ is non 0 . Let $r_{i+1}$ be the part of $T \bar{r}_{i}$ that has support at $j$. It is enough to show that $\lim _{n \rightarrow \infty} \int \bar{r}_{n}=0$ and we may assume that the support of $r$ is one point, $l$. Let $\beta(l)=\lim _{n \rightarrow \infty} \int \bar{r}_{n}$ when the support of $r=l$. $\int \sum_{i=1}^{\infty} r_{i}=1-\beta(l)$. Since $\left(\sum_{i=1}^{\infty} T \delta_{j}\right)(j)=\infty$, it is easily seen that $\beta(j)=0$. Since $\left(\sum_{i=1}^{\infty} T^{i} \delta_{j}\right)(l) \neq 0, \beta(j)=0$ implies that $\beta(l)=0$.]

(4) (2) and (3) imply that we can get a version $\tilde{g}$ of $g$ such that $\tilde{g} \geqq \gamma g+s$ where $\gamma>(1-\epsilon)^{2}$. ( $s$ is defined in (2).)

(5) It follows from (4), since $\epsilon$ could be made arbitrarily small, that for any large number $M$ we could find a version $\tilde{g}$ of $g$ such that $\tilde{g}>M s^{\prime}$ where $s^{\prime}$ has the property that $\lim _{i \rightarrow \infty} \int T^{i} s^{\prime} d u>\alpha$ large enough.

(6) It follows from (5) that $\int T^{K} \tilde{g} d u>\frac{1}{2} M \alpha$ for all $K$. Using remark (A), if $K$ is large enough we could write $T^{K} \tilde{g}=\sum_{i=1}^{n} T^{i}\left(T^{K-i} g_{i}\right)$ $+T^{n}\left(T^{K-n} \bar{g}_{n}\right)$ where $\tilde{g}=\sum_{i=1}^{n} g_{i}+\bar{g}_{n}$ and $T^{K} g=\sum_{i=1}^{n} T^{K-i} g_{i}+T^{K-n} \bar{g}_{n}$. If sup $\left\|T^{n}\right\|_{1}=B$ this shows that $\int T^{K} g d u>\frac{1}{2} M \alpha(B)^{-1}$, but since $M$ could be arbitrarily large this contradicts $\left(b^{\prime}\right)$.

Another Proof of Orey's Theorem. The same kind of argument as the one above will give another proof of Orey's theorem, and we will now indicate how to do this. (We will assume that (a)-(d) are true for the measure $u$, and we will forget about $v$.)

(1') We could, as before, assume that $f=g-h$ where $g \geqq 0, h \geqq 0$ and $g$ and $h$ have only 1 point in their support (i.e., $g=\delta_{x}, h=\delta_{y}$ ). 
(2') It is enough to show that $\lim _{n \rightarrow \infty} \int\left|T^{n} g-T^{n+1} g\right| d u=0$. To see this note that (3) tells us we can get a version $\tilde{h}$ of $h$ such that $\tilde{h}=h_{1}+\cdots+h_{n}+\bar{h}_{n}$ where each $h_{i}$ is 0 or a multiple of $g$ and $\int \bar{h}_{n} d u$ $<\epsilon .\left(2^{\prime}\right)$ now follows from Remark A.

( $\left.3^{\prime}\right)$ Given $M$ we can find $\alpha>0, n>N$, and a version $\tilde{g}$ of $g$ such that $\tilde{g}=\sum_{i=1}^{n} g_{i}+\bar{g}_{n}$ where $g_{i}=0$ for $i<n-M$ and $g_{i}=\alpha g$ for $n-M$ $\leqq i \leqq n$. This follows easily from (d).

$\left(4^{\prime}\right)$ Given $\epsilon>0$ we can find a version $\tilde{g}$ of $g$ such that $\tilde{g}=\sum_{i=1}^{m}$ $g_{i}+\bar{g}_{m}, \int \bar{g}_{m} d u<\epsilon$ and where each $g_{i}=\sum_{=1}^{l} g_{i, j}$ and for fixed $j$ all the $g_{i, j}$ are 0 except for a block of $M$ consecutive $i$ and for these $i$ all the $g_{i, j}$ are equal to the same multiple of $g$. To get $\left(4^{\prime}\right)$ we use $\left(3^{\prime}\right)$. Then apply (3) to get a version $\hat{g}$ of $\bar{g}_{n}$ where $\hat{g}=\sum_{i=1}^{k} g_{i}^{\prime}+\bar{g}_{k}^{\prime}, \int \bar{g}_{k}^{\prime} d u$ is very small and each $g_{i}^{\prime}$ is a multiple of $g$. We next apply this whole process to each $g_{i}^{\prime}$, etc.

$\left(5^{\prime}\right)$ Let $\tilde{g}$ be the version of $g$ constructed in $\left(4^{\prime}\right)$. By Remark A, $T^{K-1} g=\sum_{i=1}^{m} T^{K-i} g_{i}+T^{K-m} \bar{g}_{m}$ and hence

$$
\begin{aligned}
T^{K-1} g & =\sum_{j=1}^{l}\left(\sum_{i=1}^{m} T^{K-i} g_{i, j}\right)+T^{K-m} \bar{g}_{m}, \\
T^{K} g & =\sum_{j=1}^{l}\left(\sum_{i=1}^{m} T^{K-i+1} g_{i, j}\right)+T^{K-m+1} \bar{g}_{m} \\
& =\sum_{j=1}^{l}\left(\sum_{i=1}^{m} T^{K-i} g_{i-1, j}\right)+T^{K-m+1} \bar{g}_{m} .
\end{aligned}
$$

We now use the fact that for fixed $j, g_{i-1, j}=g_{i, j}$, except for 2 values of $i$. This shows that $\int\left|T^{K} g-T^{K-1} g\right| \leqq(2 / M) \int g+2 \epsilon$. This gives (2') and therefore Orey's theorem.

\section{BIBLIOGRAPHY}

1. Steven Orey, An ergodic theorem for Markov chains, Z. Wahrscheinlichkeitstheorie und Verw. Gebeite 1 (1962), 174-176.

2. Louis Sucheston, On the ergodic theorem for positive operators. I, Z. Wahrscheinlichkeitstheorie und Verw. Gebeite 8 (1967), 1-11. Also Bull. Amer. Math. Soc. 73 (1967), 403-406.

STANFORD UNIVERSITY 